\title{
22q12.3 microduplication overlapping the $L A R G E$ gene as a male-only affected loci responsible for increasing the risk of autism spectrum disorder
}

\author{
XUAN HUANG $^{1 *}$, YINGJUN XIE ${ }^{2 *}$ and QUN FANG ${ }^{1}$ \\ ${ }^{1}$ Department of Obstetrics and Gynecology, First Affiliated Hospital of Sun Yat-sen University, Guangzhou, \\ Guangdong 510080; ${ }^{2}$ Key Laboratory for Major Obstetric Diseases of Guangdong, Key Laboratory of \\ Reproduction and Genetics of Guangdong Higher Education Institutes, The Third Affiliated Hospital \\ of Guangzhou Medical University, Guangzhou, Guangdong 510150, P.R. China
}

Received April 18, 2017; Accepted May 26, 2017

DOI: $10.3892 /$ br.2017.923

\begin{abstract}
The present study describes a three-generation Chinese family with one male who was diagnosed with an autism spectrum disorder (ASD) disease. The male proband presented with features of an autism spectrum disorder. Magnetic resonance imaging demonstrated an abnormal high-intensity zone in the frontal white matter. Whole-genome single nucleotide polymorphism-microarray demonstrated an interstitial 575-kb duplication of chromosome 22p12.3 that involved the LARGE gene among the six family members, which included three healthy female carriers, the affected boy and two male fetuses. Fluorescence in situ hybridization analysis, using special probes, and $L A R G E$ gene sequencing were performed, which exhibited a submicroscopic 22q13 duplication that involved the LARGE gene. Combined with a review of the literature, the present findings support the hypothesis that the $22 \mathrm{q} 12.3$ microduplication overlapping the $L A R G E$ gene may be a male-only affected loci, which is responsible for increasing the ASD risk.
\end{abstract}

\section{Introduction}

Autism spectrum disorder (ASD) is a group of neurodevelopmental disorders characterized by disturbances in interpersonal relationships and behavior, and is characterized by severe and pervasive impairment in reciprocal socialization, qualitative impairment in communication, and repetitive or

Correspondence to: Dr Qun Fang, Department of Obstetrics and Gynecology, First Affiliated Hospital of Sun Yat-sen University, 58 Zhongshan Road 2, Guangzhou, Guangdong 510080, P.R. China E-mail: fang_qun@163.com

\section{${ }^{*}$ Contributed equally}

Key words: microduplication, $L A R G E$ gene, copy number variation, autism spectrum disorder unusual behavior (1). It is also known that ASD is male-biased and present genetic heterogeneity (2). However, it is difficult to identify the common causes in this heterogeneous disorder. Previous genome-wide association studies have identified numerous mutations or variations associated with ASD risk on many chromosome loci and genes $(1,3,4)$. Furthermore, a promising development in understanding the genetics of ASD is the identification of variations in the gene copy number as a risk factor (5).

Copy-number variation is defined as the structural variation in the genome in which genetic material is either duplicated or deleted and appears to be strongly associated with various nervous system disorders and mental illnesses, such as intellectual impairment and autism $(6,7)$. While sequencing of sporadic cases has identified de novo risk variants, the heritable genetic contribution and mechanisms driving the male bias are complex and less understood $(2,8,9)$. A previous study highlighted the male-specific effect of the rs6323 marker and its haplotypes in ASD etiology, revealing the sexual dimorphic effect of monoamine oxidase A (responsible for degradation of serotonin) in ASD (1). From a total sample of 1,008 multiplex families, Werling et al (10) performed genome-wide, non-parametric linkage analysis in a discovery sample of 847 families separated by subsets of families with only male affected children (male-only; MO) or with at least one female affected child (female-containing; FC), and observed sex-differential linkage at 1p31.3 (MO), 8p21.2 (FC), and 8p12 (FC).

The current study describes a three-generation Chinese family (Fig. 1A) with one affected male, who was diagnosed with an ASD disease using whole-genome single nucleotide polymorphism (SNP)-microarray and fluorescence in situ hybridization analysis, with special probes, and determined a 22q12.3 duplication among the six family members, which included three healthy female carriers, one affected boy and two male fetuses. In addition, $L A R G E$ gene sequencing indicated that the $22 \mathrm{q} 12.3$ duplication overlapped a $L A R G E$ gene. The present study hypothesized that the specific $22 \mathrm{q} 12.3$ duplication overlapping the $L A R G E$ gene may be a male-only loci, which is responsible for increasing the nervous system disorder risk, including ASD. 


\section{Materials and methods}

Clinical investigation of the patient and family. A 35-year-old woman (II:2), gravida 4 para 3, was referred to the First Affiliated Hospital of Sun Yat-sen University (Guangzhou, China) for genetic consultation at gestational week 28 in March 2013, due to detection of fetal cerebellar vermis by prenatal ultrasound at gestational week 27. A fetal ultrasound demonstrated that the cerebellar vermis area was $1.63 \mathrm{~cm}^{2}$ $\left(2.27 \pm 0.64 \mathrm{~cm}^{2}\right)$ and polyhydramnios was observed (amniotic fluid volume, $98 \mathrm{~mm}$ ). Additionally, fetal magnetic resonance imaging (MRI) indicated that fetal brain development was significantly slower than its gestational age, the hemispheric sulci and gyri were not obvious and lateral cerebral fissure was shallow. Her husband (II:1) was 42 years old. The couple was non-consanguineous and had no family history of congenital malformations on either side.

However, the couple had an abnormal childbearing history. The first child (III:1) of the couple was a boy who was diagnosed with ASD. At the time of the study, the proband was 9 years old. He was delivered normally (birth weight, $3.2 \mathrm{~kg}$ ); however, the birth head circumference was not available. Mild hypospadias was noted. At 30 months of age, he showed microcephaly, social deficits and communication difficulties, stereotyped or repetitive behaviors and interests, sensory issues, and was diagnosed as ASD. He was unable to walk until the age of 4 with marked motor and language delay. He had a long face, periorbital fullness, a smooth philtrum, and a thin upper lip vermilion. He also had tapering fingers and camptodactyly. He was characterized by frequent mood swings and difficulties obeying rules. At the age of 7, brain MRI indicated a small brain size, focal radial distribution of defects on the front parts of the two frontal lobes, the right side of the Brota area and the left side of the hippocampus.

The second child (III:2) of the couple wasa 4-year-old healthy girl. The third sibling was a male fetus. Ultrasound-guided amniocentesis of the fetuses (III:3 and III:4) was performed for a cytogenetic analysis and chromosomal microarray analysis (CMA) was performed at gestational week 18. The CMA result was as abnormal as the proband. Therefore, subsequent to receiving genetic counseling, the parents decided to terminate the third pregnancy.

Umbilical cordocentesis was then performed at gestation week 28. Umbilical cord blood was collected for cytogenetic analysis and CMA. Due to the malformation of the fetal brain and abnormal CMA result, the parents opted to have an abortion. The pregnancy was subsequently terminated, and a male fetus was delivered. However, the brain malformation could not be determined, as the parents did not consent to fetal autopsy.

Approval was obtained for the current study from the Ethics Committee of the First Affiliated Hospital of Sun Yat-sen University (Guangzhou, China). Principles outlined in the Declaration of Helsinki were followed and informed consent was obtained from the participants.

Cytogenetics and SNP array analyses. Cytogenetic analysis was performed on G-banded metaphases at a resolution of 450-550 bands. Chromosome preparations were established from cultured lymphocytes of the proband and the couple.
Table I. Primer sequences for sequencing of $L A R G E$ gene.

\begin{tabular}{ll}
\hline Primer & \multicolumn{1}{c}{ Sequence $\left(5^{\prime}-3^{\prime}\right)$} \\
\hline 1 & F:GCTGTGTGTAAGTGTGTTTTATATC \\
& R:GCAAGCCAGTGGAGAG \\
2 & F:GTTTACGCCTCATGGATTTA \\
& R:GGGCACACAGTCCCAA \\
3 & F:TCAAAGACCCATATCAACCA \\
& R:GTGCTGAAAAGCGACACTTA \\
& F:AGCTTCATACACTGAAATTGTTG \\
5 & R:CACCGGGAAACCTTGAT \\
& F:CTCTGAGACCACTTTCAAGC \\
6 & R:ATGTTACCCATTTGTGGAGA \\
& F:AGTACCTGAAAGGGTGGG \\
7 & R:GATCAGTGTAGGCTCCAAA \\
& F:CTTAATGGTTTGGCAGGATA \\
& R:AGCTTAAAANCAAAATCTCCC
\end{tabular}

F, forward primer; $\mathrm{R}$, reverse primer.

Peripheral venous blood $(2 \mathrm{ml})$ was collected from the couples, their two children, the sister of the mother and the mother's mother, and cord blood was collected from the present fetus (the fourth sibling). Genomic DNA was extracted from the uncultured blood samples using a QIAamp DNA Blood Mini kit (cat. no. 51104; Qiagen, Inc., Valencia, CA, USA). DNA (250 ng) was amplified (CytoScan PCR program), labeled and hybridized to the CytoScan HD array platform (Affymetrix; Thermo Fisher Scientific, Inc., Waltham, MA, USA) according to the manufacturer's protocol. The array is designed specifically for cytogenetic research, which offers $>2,700,000$ markers across the whole genome, including 750,000 SNP probes and $1,950,000$ probes for detecting the copy number variations (Cyto-arrays). CEL files obtained by scanning CytoScan arrays were analyzed with Chromosome Analysis Suite software (ChAS 2.1, Affymetrix), using annotations of genome version GRCH37 (hg19). Only those qualified measures were included in the present analysis. Gains and losses that affected a minimum of 50 markers in a 100-kb length were initially considered.

Fluorescence in situ hybridization (FISH). FISH was performed according to standard protocols. Single copy DNA probes (Agilent Technologies, Inc., Santa Clara, CA, USA), $22 \mathrm{q} 12.3(561 \mathrm{k})$ located within the duplication region on 22q12.3, and the commercially available probe LSI 22 (Abbott $\mathrm{GmbH} \& \mathrm{Co}$. KG, Wiesbaden, Germany) served as a chromosome 22 control probe.

Quantitative polymerase chain reaction ( $q P C R$ ). Confirmation of the CMA result and analysis of the unaffected sisters and the parents were performed by qPCR with Genomic DNA extracted from the uncultured blood samples. qPCR experiments were conducted on an ABI Prism 9700TH Sequence Detection System (Applied Biosystems; Thermo Fisher Scientific, Inc., Waltham, MA, USA) using SYBR ${ }^{\mathrm{TM}}$ Green PCR Master Mix (Applied Biosystems; Thermo Fisher 

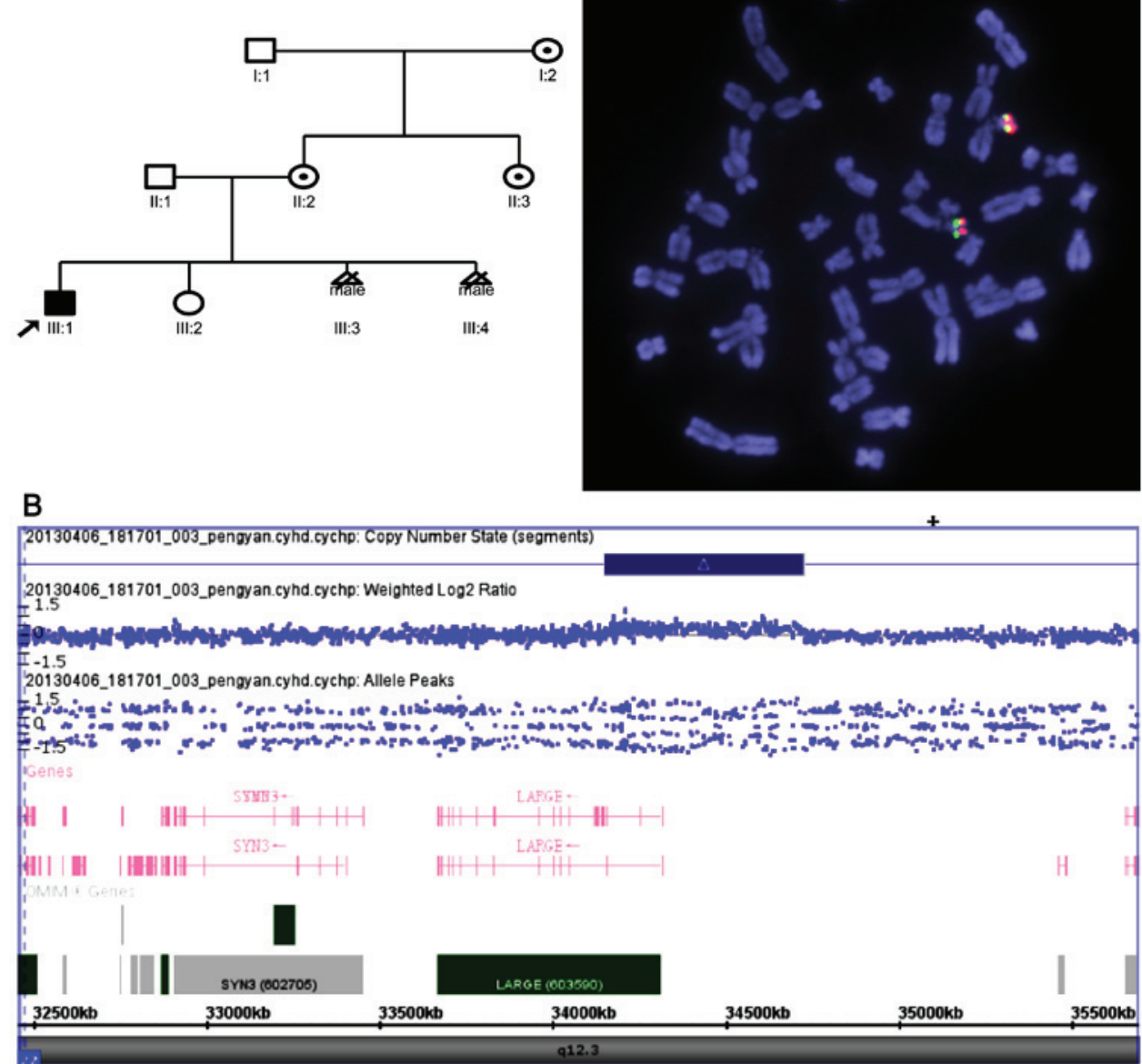

Figure 1. (A) Pedigree of a Chinese family with autism. A filled square indicates an affected male and a dot in the middle of the circle indicates a female carrier. A triangle with an oblique line indicates a therapeutic abortion. The arrow indicates the proband. (B) SNP-Array genotyping of patients. Whole-genome array-based SNP demonstrated a 575-kb duplication stretching from 34,151,455 to 34,726,809 bp. (C) A partial map of the $22 \mathrm{q} 12.3$ region demonstrating the location of the fluorescence in situ hybridization probes and genes in the region.

Scientific, Inc.). The authors selected non-polymorphic fragments locating in the LARGE gene. The used primers for qPCR were as follow: Forward, 5'-CAACCACTCCAAGAC CTACTC-3' and reverse, 5'-CGCATTTCTCCACGACCG-3'. GADPH was used as internal reference (forward, GGAGTC AACGGATTTGGTCG and reverse, TCCTGGAAGATG GTGATGGG) and the reaction conditions were as follows: $95^{\circ} \mathrm{C}$ for $10 \mathrm{~min}, 40 \mathrm{cycles}$ of $95^{\circ} \mathrm{C}$ for $10 \mathrm{sec}, 60^{\circ} \mathrm{C}$ for $30 \mathrm{sec}$ and $72^{\circ} \mathrm{C}$ for $30 \mathrm{sec}$ ). The resultant crossing thresholds were analyzed using the $\mathrm{Cq}$ method. The final data were compared with a normal control in the same loci using $2^{-\Delta \Delta C q}$ (11).

$L A R G E$ gene sequencing. Overlapping amplicons covering the entire coding region of 15 exons from all isoforms of $L A R G E$ (MIM no. 603590) were amplified. PCR was performed in 10 reactions using $100 \mathrm{ng}$ genomic DNA or $1 \mathrm{ng}$ cloned DNA in standard buffer (Boehringer Mannheim GmbH, Mannheim, Germany): $10 \mathrm{mM}$ Tris- $\mathrm{HCl}, 1.5 \mathrm{mM} \mathrm{Mg}{ }^{2+}, 50 \mathrm{mM} \mathrm{KCl}$ (pH 8.3), with 0.5 U Taq polymerase (Boehringer Mannheim $\mathrm{GmbH}$, Mannheim, Germany) and $2 \mathrm{nM}$ primers. Templates were denatured at $95^{\circ} \mathrm{C}$ for $3 \mathrm{~min}$, followed by 12 cycles of $95^{\circ} \mathrm{C}$ for $2 \mathrm{~min}, 65^{\circ} \mathrm{C}$ for $40 \mathrm{sec}$, and $72^{\circ} \mathrm{C}$ for $1 \mathrm{~min}$, then followed by 45 cycles of $95^{\circ} \mathrm{C}$ for $2 \mathrm{~min}, 55^{\circ} \mathrm{C}$ for $40 \mathrm{sec}$, and $72^{\circ} \mathrm{C}$ for
$1 \mathrm{~min}$. A final extension was performed for $10 \mathrm{~min}$ at $72^{\circ} \mathrm{C}$. Reactions were performed using a Perkin Elmer 9600 thermal cycler. Individual primer sequences were listed in Table I. PCR products were analyzed using gel electrophoresis with $1.5 \%$ agarose. PCR products for sequencing were isolated from low melting point agarose using the Spinbind purification kit (FMC Bioproducts, Rockland, ME, USA), and sequenced in two directions using a BigDye Terminator v3.1 Cycle Sequencing kit (cat. no. 4337455; Applied Biosystems; Thermo Fisher Scientific, Inc.) and an ABI 3100 Genetic Analyzer (Applied Biosystems; Thermo Fisher Scientific, Inc.). DNA sequences were analyzed by comparison to GRCh37.

\section{Results}

Cytogenetic investigations performed on peripheral blood lymphocytes in all members of the family exhibited a normal karyotype. Genome-wide array analysis revealed an interstitial 575-kb duplication of chromosome 22p12.3 from 34,151,455 to $34,726,809$ bp (Fig. 1B). FISH analysis of the proband and his parents confirmed the rearrangements and excluded translocation with other chromosomes at this duplication region (Fig. 1C). qPCR performed on the gene within the borders of 
the identified duplication evidenced the overlap of the $L A R G E$ gene (intron 1). However, no significant mutations were identified by sequencing of the LARGE gene.

\section{Discussion}

The current study describes a three-generation Chinese family (six members) with an interstitial $575-\mathrm{kb}$ duplication of chromosome 22p12.3 that involved the LARGE gene. The family comprised three healthy female carriers and three affected males, including a 9-year-old boy and two male fetuses. In addition, in the Decipher database (https://decipher.sanger.ac.uk/) numerous patients exhibited overlapping duplication on chromosome 22q12.3 similar to the current patient. Patient no. 290286 had a $630-\mathrm{kb}$ duplication at chr22:33,440,702-34,073,409 and exhibited a phenotype of global developmental delay and delayed gross motor development. In addition, complex rearrangement or duplication may cause gene mutations, which lead to cognitive disorders, such as ASD if such genes are important in the development and modulation of synaptic connectivity (12). While sequencing the 15 exons of this gene did not identify any significant mutations, it is suggested that the duplication associated with ASD is more frequent in males.

The common $575-\mathrm{kb}$ duplication interval of $22 \mathrm{q} 12.3$ observed in these patients includes only one known gene, $L A R G E$ (MIM no. 603590) and the breakpoint between exon 1 and exon 2 . The duplication region overlapped the 5 ' untranslated region (5'UTR) within exon 1 of the $L A R G E$ gene. $L A R G E$ encodes one of a number of proteins that are critical in the development of the neuromuscular junction. The encoded protein contains laminin G, Kazal-type serine protease inhibitor and epidermal growth factor domains. Additional post-translational modifications occur to add glycosaminoglycans and disulfide bonds. Mutation of the LARGE gene is the rarest of the six known genetic causes (protein O-mannosyltransferase 1, protein O-mannosyltransferase 2, $\mathrm{O}$-mannose $\beta-1,2-\mathrm{N}$-acetylglucosaminyltransferase, Fukuyama type congenital muscular dystrophy protein, Fukutin-related protein and $L A R G E$ ) of $\alpha$-dystroglycanopathy $(\alpha D G)$. Abnormality in the glycosylation of $\alpha D G$ is the hallmark histological abnormality and the likely pathogenic mechanism for a group of congenital muscular dystrophies, collectively termed $\alpha$ DGs (13). Affected children exhibit typical neurological and muscular abnormalities associated with the $\alpha \mathrm{DGs}$, but with very different severities. For example, one family member had mild muscle-eye-brain disease (and the other two had typical Walker-Warburg syndrome (WWS). In addition, a WWS patient has been reported to have a single heterozygous nonsense mutation in $\operatorname{LARGE}(14,15)$. In one family with congenital myasthenic syndrome affecting limb-girdle muscles, a mutation in LARGE was observed (16). Patients with WWS frequently demonstrate a complete lack of psychomotor development, severe eye malformations, cobblestone lissencephaly and a hypoplastic cerebellum and brainstem, seizures, hydrocephalus and poor prognosis (17). Previously, Clarke et al (18) described a family with mental retardation, and identified a large intra-chromosomal duplication inserted into intron 10 of $L A R G E$ in a homozygous state. As a result, it is proposed that mutations of the $L A R G E$ gene may cause a wide spectrum of clinical phenotypes. In the patient investigated in the current study, disruption of the $L A R G E$ gene was anticipated to result in a different protein and may account for ASD and associated clinical features in the patient, as it is highly expressed in the brain.

The current results imply high diversity of clinical presentation as well as sex-bias, such as the three normal females and three affected males in the present study. The high diversity of clinical presentation may also be caused by the influence of interactions between genetic and environmental factors on clinical manifestation or differential environmental exposures experienced by different individuals $(19,20)$. As for the striking sex-bias in autism, early exposure to androgenic hormones and early maternal immune activation are environmental factors, and have been proposed to affect the sex-specific susceptibility to ASD (21). Baron-Cohen's hypothesis that autism results from exposure to high intrauterine testosterone levels is considered in the context of a hormonal hypothesis of sex ratio and the notion of multifactorial inheritance (22). This hypothesis yields three suggestions as follows: i) Female cases of autism may be the product of higher genetic loading combined with moderate environmental exposure and male cases of high environmental exposure combined with moderate genetic loading; ii) notably, one of the environmental agents is intrauterine testosterone; and iii) the mother is the major source of that testosterone. A gender-atypical pattern for these types of feature is indicated in ASD (23). These suggestions may help to explain the majority of the major established epidemiological risk factors for autism.

In conclusion, the present study identified a role for 22q12.3 duplication in the diversity of phenotypes with ASD and brain deformation in patients exhibiting a 22q12.3 duplication. In addition, it is hypothesized, but as yet unproven, that the specific 22q12.3 duplication overlapping the $L A R G E$ gene may be within the male-only loci that are responsible for increasing ASD risk. However, further in-depth evaluation of other ASD patients with defects in the $L A R G E$ gene are required to elucidate the genetic mechanism underlying ASD.

\section{Acknowledgements}

The authors would like to thank the Clinical Cytogenetics Laboratory (the First Affiliated Hospital of Sun Yat-sen University, Guangzhou, China) for facilitating with data collection. The authors would also like to thank the individuals included in the current study and their families.

\section{References}

1. Cho SC, Yim SH, Yoo HK, Kim MY, Jung GY, Shin GW, Kim BN, Hwang JW, Kang JJ, Kim TM, et al: Copy number variations associated with idiopathic autism identified by whole-genome microarray-based comparative genomic hybridization. Psychiatr Genet 19: 177-185, 2009.

2. Horiuchi F, Oka Y, Uno H, Kawabe K, Okada F, Saito I, Tanigawa T and Ueno S: Age- and sex-related emotional and behavioral problems in children with autism spectrum disorders: Comparison with control children. Psychiatry Clin Neurosci 68: 542-550, 2014.

3. Rosenfeld JA, Ballif BC, Torchia BS, Sahoo T, Ravnan JB, Schultz R, Lamb A, Bejjani BA and Shaffer LG: Copy number variations associated with autism spectrum disorders contribute to a spectrum of neurodevelopmental disorders. Genet Med 12: 694-702, 2010. 
4. GriswoldAJ,MaD,CukierHN,NationsLD,SchmidtMA,ChungRH, Jaworski JM, Salyakina D, Konidari I, Whitehead PL, et al: Evaluation of copy number variations reveals novel candidate genes in autism spectrum disorder-associated pathways. Hum Mol Genet 21: 3513-3523, 2012.

5. Sebat J, Lakshmi B, Malhotra D, Troge J, Lese-Martin C, Walsh T, Yamrom B, Yoon S, Krasnitz A, Kendall J, et al: Strong association of de novo copy number mutations with autism. Science 316: 445-449, 2007.

6. Cook EHJ Jr and Scherer SW: Copy-number variations associated with neuropsychiatric conditions. Nature 455: 919-923, 2008.

7. Prasad A, Merico D, Thiruvahindrapuram B, Wei J, Lionel AC, Sato D, Rickaby J, Lu C, Szatmari P, Roberts W, et al: A discovery resource of rare copy number variations in individuals with autism spectrum disorder. G3 (Bethesda) 2: 1665-1685, 2012.

8. Verma D, Chakraborti B, Karmakar A, Bandyopadhyay T, Singh AS, Sinha S, Chatterjee A, Ghosh S, Mohanakumar KP, Mukhopadhyay K, et al: Sexual dimorphic effect in the genetic association of monoamine oxidase A (MAOA) markers with autism spectrum disorder. Prog Neuropsychopharmacol Biol Psychiatry 50: 11-20, 2014.

9. Mouridsen SE, Rich B and Isager T: The Sex Ratio of Full and Half Siblings of People Diagnosed With ADHD in Childhood and Adolescence: A Danish Nationwide Register-Based Cohort Study. J Atten Disord 20: 1017-1022, 2016.

10. Werling DM, Lowe JK, Luo R, Cantor RM and Geschwind DH: Replication of linkage at chromosome 20p13 and identification of suggestive sex-differential risk loci for autism spectrum disorder. Mol Autism 5: 13, 2014

11. Livak KJ and Schmittgen TD: Analysis of relative gene expression data using real-time quantitative PCR and the 2(-Delta Delta C(T)) Method. Methods 25: 402-408, 2001.

12. Knight D, Xie W and Boulianne GL: Neurexins and neuroligins: Recent insights from invertebrates. Mol Neurobiol 44: 426-440, 2011.

13. Martin PT: Mechanisms of disease: Congenital muscular dystrophies-glycosylation takes center stage. Nat Clin Pract Neurol 2: 222-230, 2006
14. Mercuri E, Messina S, Bruno C, Mora M, Pegoraro E, Comi GP, D'Amico A, Aiello C, Biancheri R, Berardinelli A, et al: Congenital muscular dystrophies with defective glycosylation of dystroglycan: A population study. Neurology 72: 1802-1809, 2009.

15. Godfrey C, Clement E, Mein R, Brockington M, Smith J, Talim B, Straub V, Robb S, Quinlivan R, Feng L, et al: Refining genotype phenotype correlations in muscular dystrophies with defective glycosylation of dystroglycan. Brain 130: 2725-2735, 2007.

16. Maselli RA, Fernandez JM, Arredondo J, Navarro C, Ngo M, Beeson D, Cagney O, Williams DC, Wollmann RL, Yarov-Yarovoy $\mathrm{V}$, et al: LG2 agrin mutation causing severe congenital myasthenic syndrome mimics functional characteristics of non-neural (z-) agrin. Hum Genet 131: 1123-1135, 2012.

17. Czeschik JC, Hehr U, Hartmann B, Lüdecke HJ, Rosenbaum T, Schweiger B and Wieczorek D: $160 \mathrm{~kb}$ deletion in ISPD unmasking a recessive mutation in a patient with Walker-Warburg syndrome. Eur J Med Genet 56: 689-694, 2013.

18. Clarke NF, Maugenre S, Vandebrouck A, Urtizberea JA, Willer T, Peat RA, Gray F, Bouchet C, Manya H, Vuillaumier-Barrot S, et al: Congenital muscular dystrophy type 1D (MDC1D) due to a large intragenic insertion/deletion, involving intron 10 of the LARGE gene. Eur J Hum Genet 19: 452-457, 2011.

19. Lai MC, Lombardo MV and Baron-Cohen S: Autism. Lancet 383: 896-910, 2014.

20. Cuccaro ML, Shao Y, Bass MP, Abramson RK, Ravan SA, Wright HH, Wolpert CM, Donnelly SL and Pericak-Vance MA: Behavioral comparisons in autistic individuals from multiplex and singleton families. J Autism Dev Disord 33: 87-91, 2003.

21. Schaafsma SM and Pfaff DW: Etiologies underlying sex differences in Autism Spectrum Disorders. Front Neuroendocrinol 35: 255-271, 2014.

22. James WH: An update on the hypothesis that one cause of autism is high intrauterine levels of testosterone of maternal origin. J Theor Biol 355: 33-39, 2014.

23. Bejerot S and Eriksson JM: Sexuality and gender role in autism spectrum disorder: A case control study. PLoS One 9: e87961, 2014. 